\title{
Feeding Versus Feedback in AGNs from Near-Infrared IFU Observations: The Case of Mrk 1066
}

\author{
Rogemar A. Riffel ${ }^{1,2}$ and Thaisa Storchi-Bergmann ${ }^{1}$ \\ ${ }^{1}$ Universidade Federal do Rio Grande do Sul, RS, Brazil \\ Email: rogemar@ufrgs.br \\ ${ }^{2}$ Universidade Federal do Santa Maria, RS, Brazil
}

Keywords. galaxies: active, galaxies: ISM, galaxies: nuclei, galaxies: jets

Previous studies of the central region of active galaxies show that the molecular and ionized gas have distinct kinematics and flux distributions, with the former dominated by quiescent kinematics characteristic of rotation in the galactic plane and the latter with more disturbed kinematics and apparently extending to larger galactic latitudes. These results suggest that the molecular gas can be a tracer of the feeding of the AGN and the ionized gas a tracer of its feedback (e.g., Riffel et al. 2009, 2008, 2006; Storchi-Bergmann et al. 2009a,b). In the present study we use Gemini NIFS integral field observations of the inner $700 \times 700 \mathrm{pc}^{2}$ of the Seyfert galaxy Mrk 1066 at a spatial resolution of $\sim 35 \mathrm{pc}$ to investigate if the above scenario is also valid for this galaxy.

The flux distributions for $\mathrm{H}_{2} \lambda 2.121 \mu \mathrm{m}$, [Fe II] $\lambda 1.257 \mu \mathrm{m}$, [P II] $\lambda 1.189 \mu \mathrm{m}, \mathrm{Br} \gamma$, and $\mathrm{Pa} \beta$ emission lines are more extended along the position angle $\mathrm{PA}=135 / 315^{\circ}$ showing good spatial correlation with previous $3.6-\mathrm{cm}$ radio continuum and [O III] images and, except for the $\mathrm{H}$ lines, are brighter to the northwest than to the southeast, in close association with a radio hot spot. This implies that at least part of the emitting gas is co-spatial with the radio outflow. The hydrogen emission is stronger to the southeast, where we find a large region of star formation. The $\mathrm{H}_{2}$ flux is more uniformly distributed and has an excitation temperature of $\sim 2100 \mathrm{~K}$. Its origin appears to be circumnuclear gas heated by X-rays from the central active nucleus. The [Fe II] emission also is consistent with X-ray heating, but its spatial correlation with the radio jet and [O III] emission indicates additional emission due to shocks produced by the radio jet.

Three components are observed in the gaseous kinematics: (i) an outflowing gas component, clearly seen in the ionized gas, and originating from the interaction of the radio jet with the emitting gas, (ii) streaming motions towards the center along the minor axis of the galaxy, which seems to feed (iii) a compact rotating disk observed in molecular hydrogen. The distinct kinematics of the molecular and ionized gas is also shown by the lower velocity dispersion values $\left(\sigma \approx 80 \mathrm{~km} \mathrm{~s}^{-1}\right.$ ) observed for the former compared to $\sigma \approx 140 \mathrm{~km} \mathrm{~s}^{-1}$ for the latter.

A detailed discussion on the emission-line flux distributions and excitation will be presented by Riffel et al. (2010), while the gaseous and stellar kinematics will be discussed by Riffel \& Storchi-Bergmann (2010, in preparation).

\section{References}

Riffel, R. A., et al. 2006, MNRAS, 373, 2

Riffel, R. A., et al. 2008, MNRAS, 385, 1129

Riffel, R. A., et al. 2009, MNRAS, 393, 783

Riffel, R. A., et al. 2010, MNRAS, in press [arXiv1001.0130]

Storchi-Bergmann, T., et al. 2009, MNRAS, 394, 1148

Storchi-Bergmann, T., et al. 2009, MNRAS, in press 